\title{
Daptomycin elimination by continuous venovenous hemofiltration: in vitro evaluation of factors influencing sieving and membrane adsorption Claudia Wagner ${ }^{1}$, Ilka Steiner ${ }^{2}$ and Markus Zeitlinger*1
}

\author{
Address: ${ }^{1}$ Department of Clinical Pharmacology, Medical University of Vienna, Austria and ${ }^{2}$ Institute of Pharmacology, Center for Biomolecular \\ Medicine and Pharmacology, Medical University of Vienna, Austria \\ Email: Markus Zeitlinger* - markus.zeitlinger@meduniwien.ac.at \\ * Corresponding author
}

from I3th Scientific Symposium of the Austrian Pharmacological Society (APHAR). Joint Meeting with the Austrian Society of Toxicology (ASTOX) and the Hungarian Society for Experimental and Clinical Pharmacology (MFT)

Vienna, Austria. 22-24 November 2007

Published: 14 November 2007

BMC Pharmacology 2007, 7(Suppl 2):A2I doi:I0.1 I86/I47I-22I0-7-S2-A2I

This abstract is available from: http://www.biomedcentral.com/I47I-22I0/7/S2/A2I

(C) 2007 Wagner et al; licensee BioMed Central Ltd.

The present in vitro study set out to determine for the first time the continuous venovenous hemofiltration $(\mathrm{CVVH})$ clearance of the novel lipopetide antibiotic, daptomycin, from human whole blood. Factors influencing daptomycin sieving and membrane adsorption were investigated in an in vitro setting. A recirculation model was established and daptomycin was added to the simulated blood circuit at different concentrations and in different solvent systems. The concentration of daptomycin over time in the modelled blood compartment and in ultrafiltrate was measured by HPLC. Mean sieving coefficients (SCs) of daptomycin over time were $1 \pm 0.05,0.3 \pm 0.02$ and $0.4 \pm$ 0.03 in Ringer lactate, Ringer lactate containing human albumin and plasma, respectively. The CVVH clearance of daptomycin from whole blood exceeded the physiological clearance in an individual with normal renal function. Adsorption of daptomycin to synthetic surfaces proved moderate, resulting in loss of around $20 \%$ of the initial dose at 1 hour after the start of CVVH. Since mean SCs of daptomycin in protein-containing media were higher than the free fraction in plasma, our results suggest that besides protein binding, unknown factors such as intracellular drug partitioning influence sieving of daptomycin during hemofiltration. Due to the high in vitro CVVH clearance of daptomycin we determined from human whole blood, we recommend monitoring of daptomycin concentrations in patients undergoing hemofiltration. 